\title{
Canal of Schlemm
}

National Cancer Institute

\section{Source}

National Cancer Institute. Canal of Schlemm. NCI Thesaurus. Code C32256.

A circular vascular-like structure in the anterior chamber of the eye through which aqueous humor passes when returning to the blood. 